American Journal of Immunology 4 (3): 26-32, 2008

ISSN 1553-619X

(C) 2008 Science Publications

\title{
Better Understanding of the Immunosuppressive Link between the Lymphocytic Immune Cells and the Decreased Cell Mediated Immunity in Head and Neck Cancer Patients
}

\author{
${ }^{1}$ A.S. Abdulamir, ${ }^{2}$ R.R. Hafidh ${ }^{3}$ N. Abdulmuhaimen, ${ }^{4}$ F. Abubaker and ${ }^{4}$ K.A. Abbas \\ ${ }^{1}$ Microbiology Research Department, UPM, Malaysia \\ ${ }^{2}$ Institute of Bioscience, UPM, Malaysia \\ ${ }^{3}$ College of Medicine, Alnahrain University, Iraq \\ ${ }^{4}$ Faculty of Food Science and Technology, UPM, Malaysia
}

\begin{abstract}
The purpose of this study was to determine the phenotyping of Peripheral Blood Lymphocytes (PBL) in Head and Neck Cancers (HNCA) patients and to relate this with the level of Cell-Mediated Immunity (CMI) measured by in vitro lymphoproliferative assay, in order to evaluate immune suppression in HNCA patients and its possible mechanisms. Accordingly, one hundred twenty two HNCA patients and 100 control subjects were enrolled in this study. HNCA patients were classified into 42 nasopharyngeal carcinoma, 66 carcinoma of larynx and 14 Hypo Pharyngeal Carcinoma (HPC). For measuring CMI, Microculture Tetrazolium assay (MTT) was applied on the freshly isolated lymphocytes of HNCA patients and control group. Immunophenotyping of PBL was carried out for monitoring the blood level of CD3+, CD4+, CD8+, CD21+ cells in HNCA patients in comparison with controls. The results of both assays have been integrated, revealed the presence of remarked immune suppression in HNCA patients in comparison with the controls, especially for NasoPharyngeal Carcinoma (NPC) patients who were immunosuppressed more than other studied HNCA types. Surprisingly, NPC group showed the lowest CMI level along with the highest level of PBL subsets, particularly NPC patients expressed the highest level of CD8+ cells among HNCA. It was inferred that $\mathrm{CD} 8+$ cells were more likely immune suppressor rather than cytotoxic cells and this is the principal factor for inducing sustained immunosuppression in HNCA and in NPC in particular. Furthermore CD4/CD8 ratio proved to be a reliable index for assessing the immunological status of HNCA patients and more dependable index than other immunity-evaluating factors.
\end{abstract}

Key words: Head and neck cancer, HNCA, nasopharyngeal carcinoma, NPC, immunosuppression

\section{INTRODUCTION}

Cancers in general are susceptible to immunemediated destruction and has been shown that many cancer patients have defects in their cellular immunological function, this is especially characteristic of those with HNCA that is strictly related to viral origin, Epstein Barr Virus (EBV) ${ }^{[1]}$. EBV infects more than $90 \%$ of the human adult population and is considered as the classic target for immune surveillance of persistent viral infections in humans ${ }^{[2]}$. Different EBV latency programs are associated with many important tumors, like Hodgkin's disease and nasopharyngeal carcinoma and also the EBVtransformed B cell lines (lymphoblastoid cell lines) that are so often used in human immunological research ${ }^{[3]}$.

In vitro lymphocyte proliferate/cytotoxic reactions are widely used for the detection of CMI potentials ${ }^{[4]}$.
One of the most prevalent in vitro methods have been used to test CMI function is the 3-[4,5, dimethylthiazol2-yl]-2, 5diphenyl tetrazolium bromide microculture assay (MTT) ${ }^{[5]}$.

Peripheral Blood Lymphocytes (PBL) can give a reliable monitoring of the immunological status in patients with cancers ${ }^{[6]}$. PBL CD markering is one of the main approaches that has been used to clarify the lines of immune defense mechanisms that exert an active role in the defense against tumor cells of $\mathrm{HNCA}^{[7]}$. It has been established that CD8+ cells are of a role in inducing immune suppression state in HNCA patients $^{[8,9]}$.

There was no previous study showed clearly the interlink between the lymphocytic immune cells in the immunosuprressed HNCA patients and the remarkable decline of CMI. Moreover, because that there is a remarkable geographical variation in the incidence of

Corresponding Author: Kassim A. Abbas, Department of Food Technology, Faculty of Food Science and Technology, UPM, Malaysia Tel:+603-89418534 Fax+603-89423552 
HNCA types ${ }^{[10]}$ and there was no previous immunological study on HNCA in the Middle East region, therefore it was intended in this study to evaluate the cell-mediated immunity in NPC and other types of HNCA patients in comparison with controls, integrating the results with that of the PBL immunophenotyping, in order to determine; the level of immunosuppression in HNCA, comparing the level of that suppression among different HNCA groups and determine which immunological line and which PBL subset is more associated with the induction of immunosuppression.

\section{MATERIALS AND METHODS}

One hundred twenty two Head and Neck Cancer (HNCA) patients were selected without any bias to certain type of HNCA in the period between January 2006-Dec 2007 from two main centers; University hospital of The Medical College of Alnahrain University, The Radiotherapy Reference Center in Baghdad, Iraq and Alhussein Hospital in Amman, Jordan. The samples processing and the study were totally conducted in Univeristy Putra Malaysia (UPM) in Kuala Lumpur. HNCA patients were selected preoperatively and after the diagnosis was established, primary HNCA was only selected rather than secondary or recurrent cases. One hundred of age and sex matched control subjects were selected. Control subjects were those who attended the University hospital of The Medical College of Alnahrain University for minor traumatic therapy. They showed normal blood and biochemical laboratory examinations for traumatic surgical interventions. The groups of HNCA studied were 42 patients of NPC, 66 patients of laryngeal cancer and 14 patients of hypopharyngeal carcinoma. Other HNCA types were of small numbers that were neglected for statistical analysis. Blood samples were taken from HNCA patients and from controls after obtaining full written consent. Withdrawn blood was held in heparinized tubes for later isolation of PBL.

\section{Cell mediated immunity determination:}

Lymphocytes isolation: Lymphocytes isolation from heparin zed whole blood was used in order to prepare a pure population of lymphocytes to be used in MTT assay.

Lymphocyte separation was done inside an ultraviolet hood. The procedure was based on the gradient density sedimentation technique by using Ficoll hypaque. Diluted blood (1: 2) with RPMI 1640 media was added slowly onto below Ficoll layer to create two interfacing layers. By the effect of centrifugal force, PBL were grouped into web-like layer to be withdrawn and washed later. Final concentration of lymphocytes suspension was $1-2 \times 10^{6}$ cells- $\mathrm{mL}^{-1[11]}$.

MTT assay: By MTT assay we can measure the proliferative activity of living cells when exposed to mitogenic substances ${ }^{[12]}$. In this study, we used MTT assay on the isolated PBL suspensions.

MTT assay was applied by using $100 \mu \mathrm{L}$ of final PBL suspension added to each well in duplicates. Cells were cultured in humid sterile incubator for 1 day, then $10 \mu \mathrm{L}$ of the mitogen Con A were added at concentration of $40 \mu \mathrm{g} \mathrm{mL} \mathrm{m}^{-1}$. Incubation for 3 days in incubator at $37^{\circ} \mathrm{C}$. At the 4 th day MTT agent was added at $5 \mathrm{mg} \mathrm{mL}$ with incubation for $4 \mathrm{~h}$. Finally, the supernatant was removed in each well by Pasteur pipette and $100 \mu \mathrm{L}$ of isopropanol was added, then reading by ELISA reader at $540 \mathrm{~nm}^{[13]}$.

The CMI level was measured relying on certain parameter that represents the mitogenic reactivity of PBL in MTT assay, which is called the proliferative percentage and according to the following equation ${ }^{[13]}$ :

$$
\text { Proliferative } \%=\left[\frac{\text { absorbency of experimental wells }}{\text { absorbency of control wells }}\right]-1 \times 100
$$

\section{Immunophenotyping of Peripheral blood lymphocytes:}

Fixation step: The fixation procedure dissolves and removes some of lipids, so that all of the cellular proteins, both surface and intracellular and nucleic acids were being accessible to the added antibodies. In addition, fixation is critical to the cell adhesion of PBL to immunofluorescence slides in order to be sticky throughout the later washing steps ${ }^{[14]}$.

Fifteen ul of final PBL suspension were added to immunofluorescence slide, drying at room temperature within 1-2 h. Then $15 \mu \mathrm{L}$ of the fixative Buffered Formal Acetone (BFA) was added, it was allowed to dry within $1 \mathrm{~h}$. Finally, freezing at $-20^{\circ} \mathrm{C}$.

Direct immune-fluorescence microscopy: Fifteen $\mu \mathrm{L}$ of diluted (1: 5) immunofluorescence-labeled monoclonal antibodies, namely, anti-CD3, anti-CD4, anti-CD8 and anti-CD21. Each monoclonal antibody was applied into duplicate wells. Then slides were incubated in a humid chamber at $37^{\circ} \mathrm{C}$ with mild shaking for one hour. Then, washing step was conducted for once by dipping slides into PBS-filled jar with stirring for $10 \mathrm{~min}$. One to two drops of mounting fluid were added, followed by adding cover-slips to be 
read under immunofluorescent microscope at 40 and 100x. First, at light microscopy, a suitable countable field was chosen and the number of total lymphocytes were counted, then UV light was used to count the only stained cells. The percentage of the stained cells, each represents a distinct PBL subset, was be calculated out of the total PBL cells ${ }^{[11]}$.

\section{RESULTS AND DISCUSSION}

Immune suppression featured by MTT assay: The first step for evaluating the immune status of HNCA patients was by evaluating the CMI level in HNCA patients in comparison with that of the control group by exploiting the potential of PBL to be stimulated by different mitogenic substances. The second step was to link the results of PBL CD markering with the results of CMI evaluation, in order to determine which PBL subset is more associated with immune suppression.

Proliferative percentage was used as an index for the CMI level in HNCA patients and in controls. The higher the proliferative percentage the higher the level of CMI activity would be expected and vice versa.

It was found that NPC patients had significantly the lowest proliferative percentage among all HNCA patients which was $42.5 \%$, while in laryngeal carcinoma patients the proliferative percentage was $78.7 \%$ and the highest level was in hypopharyngeal carcinoma which was $107.7 \%$. It was found that NPC patients had remarkably lower proliferative percentage than other HNCA groups $(p=0.01)$ as shown in Fig. 1 . In addition, the proliferative percentage of NPC and laryngeal carcinoma groups was much lower than that of control group, $166.7 \%(\mathrm{p}<0.00001)$, while the proliferative percentage of hypopharyngeal carcinoma, $107.7 \%$, was lower that of control group but not significantly $(p=0.07)$ as shown in Fig. 1 .

Immune suppression featured by CD markering of PBL: It was shown by PBL immunophenotyping that HNCA groups generally showed significantly lower PBL subsets than that in control group, as shown in Table 1-3. Nevertheless, NPC group showed unexpectedly a very high percentage of CD8+ cells which was even higher than that in control group. Furthermore, NPC group showed a close percentage of $\mathrm{CD} 21+$ cells with that of control group.

Therefore, NPC group showed the highest percentages of PBL subsets studied then laryngeal carcinoma group and finally hypopharyngeal carcinoma group, as shown in Fig. 2. Furthermore, CD4+/CD8+ ratio was the lowest in NPC group then in carcinoma of larynx and lastly in hypopharyngeal carcinoma, as shown in Table 4.

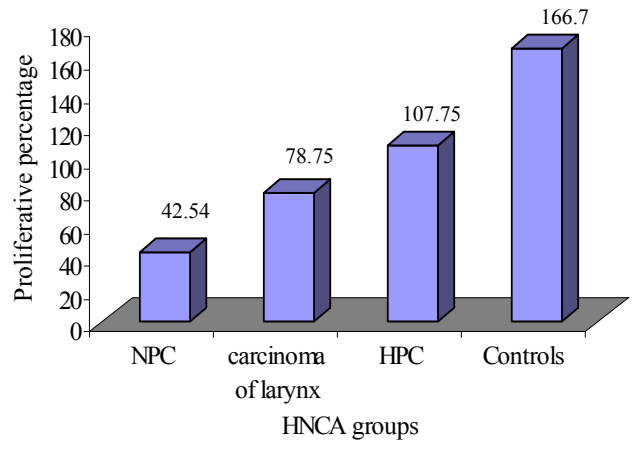

Fig. 1: The proliferative percentage (CMI level) of different HNCA and control groups

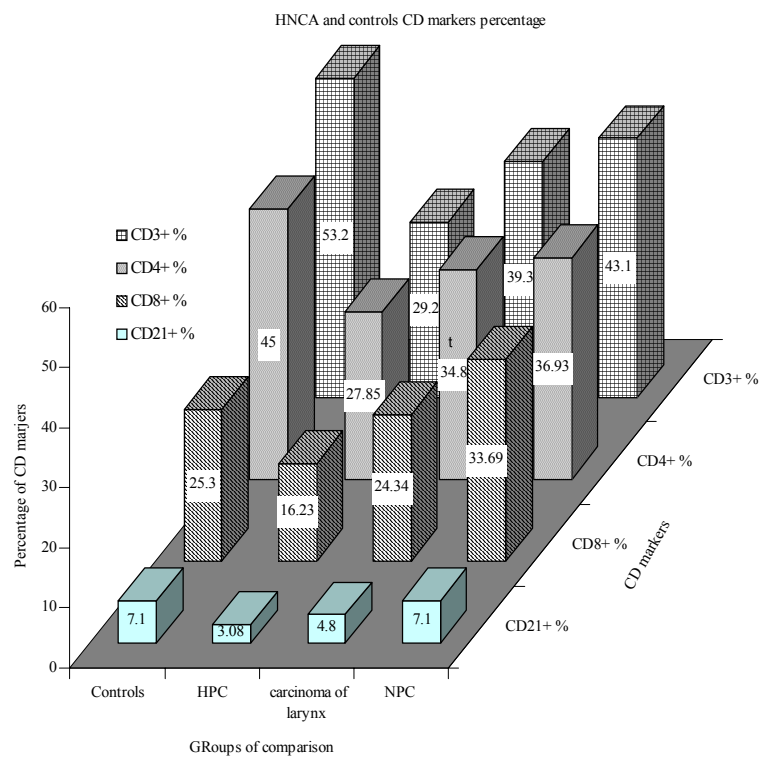

Fig. 2: Percentages of CD3+, CD4+, CD8+ and CD21+ cell populations for different HNCA and control groups

Table 1: PBL cellular expression of CD3, CD4, CD8 and CD21 in NPC patients in comparison with controls

\begin{tabular}{lll}
\hline NPC & Controls & p-value \\
\hline CD $3 \%$ & CD3\% & 0.0001 \\
43.1 & 53.2 & \\
CD $4 \%$ & CD4\% & 0.01 \\
36.93 & 45 & \\
CD $8 \%$ & CD $\%$ & 0.0002 \\
33.69 & 25.3 & \\
CD $21 \%$ & CD $21 \%$ & 1 \\
7.1 & 7.1 & \\
\hline
\end{tabular}

Table 1-3: Z-test.

Linking results of CD markering and MTT assay in HNCA patients: CMI level checked by MTT was 
Table 2: PBL cellular expression of CD3, CD4, CD8 and CD21 in CA larynx patients in comparison with controls

\begin{tabular}{lll}
\hline CA larynx & Controls & p-value \\
\hline CD3\% 30 & CD3\% & 0.00002 \\
39.35 & 53.2 & \\
CD $4 \%$ & CD4\% & 0.00009 \\
34.89 & 45 & \\
CD $8 \%$ & CD $8 \%$ & 0.1118 \\
24.34 & 25.3 & \\
CD21\% & CD21\% & 0.0003 \\
4.8 & 7.1 & \\
\hline
\end{tabular}

Table 3: PBL cellular expression of CD3, CD4, CD8 and CD21 in HPC patients in comparison with controls

\begin{tabular}{lll}
\hline HPC & Controls & p-value \\
\hline CD3 \% & CD3\% & 0.0004 \\
29.21 & 53.2 & \\
CD $4 \%$ & CD $4 \%$ & 0.00001 \\
27.85 & 45 & \\
CD $8 \%$ & CD $\%$ & 0.00008 \\
16.23 & 25.3 & \\
CD $21 \%$ & CD $21 \%$ & 0.0003 \\
3.08 & 7.1 & \\
\hline
\end{tabular}

Table 4: Multivariate t-test between HNCA patients and controls group regarding comparison of $\mathrm{CD} 4 / \mathrm{CD} 8$ ratios

\begin{tabular}{|c|c|c|c|c|}
\hline \multicolumn{2}{|l|}{ NPC } & \multicolumn{2}{|l|}{ Controls } & \multirow[b]{2}{*}{ p-value } \\
\hline Mean & Variance & Mean & Variance & \\
\hline 1.099 & 0.037 & 1.66 & 0.11 & 0.000001 \\
\hline $\begin{array}{l}\text { Carcin } \\
1.319 \\
\text { Hypop }\end{array}$ & $\begin{array}{l}\text { larynx } \\
0.186 \\
\text { eal carcinor }\end{array}$ & $\begin{array}{l}\text { controls } \\
1.66 \\
\text { controls }\end{array}$ & 0.11 & 0.040000 \\
\hline 1.8 & 0.27 & 1.66 & 0.11 & 0.200000 \\
\hline
\end{tabular}

tested for it's correlation with percentage of different PBL CD markers in order to detect any relationship that clarifies how could the immune system in HNCA patients react against tumors. By using Pearson's correlation coefficient, It was found that CMI level of HNCA patients that represented by the proliferative percentage of PBL was inversely correlated with CD8+ cell population $(\mathrm{p}<0.05)$ and marginally directly correlated with $\mathrm{CD} 4 / \mathrm{CD} 8$ ratio $(\mathrm{p}=0.051)$, while no significant correlation was obtained with $\mathrm{CD} 4+$ or CD3+ cells.

The unusual results obtained in this study were that NPC patients had the lowest in vitro lymphoproliferative stimulatory potential among HNCA patients although they had the highest percentages of the studied CD subsets of PBL. Many reports revealed that different types of HNCA have distinct patterns of CMI functions according to MTT assay, for example virally caused NPC by EBV show more unresponsiveness to mitogens than other types of

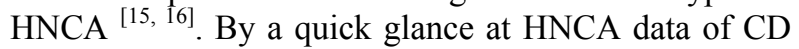
marketing, it might be confusing that there is a paradox in the results of this study. However, by linking the results of MTT assay with that of CD marketing, it was shown that the lower $\mathrm{CD} 4 / \mathrm{CD} 8$ ratio, the lower proliferative percentage (CMI level) of PBL. Consequently, the level of CD4+ cells are directly proportional to $\mathrm{CMI}$ while $\mathrm{CD} 8+$ cells are inversely proportional to CMI activity. Accordingly, it was inferred that the dominant CD8+ cells in HNCA patients were immune suppressor cells rather than cytotoxic cells. Although some reports contradict our observations in that they state the circulating CD8+ cells in NPC models do not seem to mediate nonspecific suppression of CMI and NPC patients possess high number of activated circulating cells (express HLA-DR molecule and IL-2 receptors) and a hyperproduction of IL-2 occurs when their PBL are stimulated in vitro with phytohaemagglutinin (PHA) ${ }^{[17]}$. On the other hand, some other reports revealed that CD8+ cells in HNCA patients might predominantly be of suppressor type towards T-effecter helper activity and NK cells cytotoxicity ${ }^{[8,9,18]}$.

The pivotal idea got from the PBL phenotyping was that all HNCA patients were immunosuppressed when compared with the control group. This immune suppression could be a result of the tumor itself or a cause for the initiation of carcinogenesis. This immune suppression leads to inactive immune surveillance, which is responsible for the detection of any transformed cells with attempts to eliminate them ${ }^{[19,20]}$. For the virally caused tumors, it is well known that tumor associated immune suppression is usually a subject of initial suppression of immunity that had led to virus activation and tumor development and then tumor itself would take a role in a further suppression like in NPC, Burkitt's lymphoma and Kaposi sarcoma, while other types of tumors could be triggered without a predisposing reduction in immunity ${ }^{[7]}$.

The immune suppression in HNCA patients, which was reflected by low levels of different PBL subsets, was manifested unequally among HNCA patients. In our study, NPC patients showed the highest percentages of different surface CD markers among HNCA patients. This is supported by a report ${ }^{[21]}$ which revealed that NPC patients have high levels of circulating CD3+ cells and CD4+ cells which is in agreement with our results. Interestingly, in our study, NPC patients had a very high percentage of $\mathrm{CD} 8+$ cells even higher than that of the control group, the highest percentages of PBL CD markers among the studied HNCA groups, but adversely NPC patients had the lowest CD4/CD8 ratio and the lowest CMI level among HNCA patients. This might be explained by another study revealed that EBV induced IL-10, which is strongly associated with NPC, inhibits the synthesis of IFN- $\gamma$ by lymphocytes and NK 
cells and suppresses IFN- $\gamma$-mediated cellular events such as the up-regulation of the MHC class I expression and cytotoxic cells responses leading to lower $\mathrm{CMI}^{[21]}$.

Gaining an understanding of the precise mechanism of latent EBV infection and host immune responses may contribute to promising therapeutic strategies for controlling EBV-associated disorders ${ }^{[23]}$. This study explained why NPC does not evoke a notable cytotoxic $\mathrm{T}$ lymphocytes response and the declining of EBV cytotoxic response level in advanced patients was accompanied by increase of circulating EBV DNA ${ }^{[24]}$ which is overtly a consequence of the dominance of the suppressor rather than cytotoxic CD8+ cells.

\section{CONCLUSION}

Based on the finding of the data presented here it was inferred that NPC group showed the lowest CMI level but paradoxically with the highest levels of PBL subsets, particularly NPC patients expressed the highest level of CD8+cells among HNCA. Since NPC is well known to be associated with EBV as a causative agent, consequently it was inferred that immune suppression in NPC was both a cause and consequence of viral tumorogenesis. It was also concluded that $\mathrm{CD} 8+$ cells were more likely immune suppressor rather than cytotoxic cells and this is the principal factor for inducing sustained immunosuppression in HNCA and in NPC in particular. Furthermore CD4/CD8 ratio proved to be a reliable index for assessing the immunological status of HNCA patients and more dependable index than other immunity-evaluating factors. We recommend relying on CD4/CD8 ratio and on in vitro lymphoproliferative assays as useful and adequately accurate tools for the evaluation of immunity in HNCA patients rather than PBL markers alone which could mislead researchers when are taken separately. We also recommend conducting further studies on the role of CD8+ cells in immunosuppression in other kinds of cancers.

\section{REFERENCES}

1. Hadden, J.W., J. Endicott, P. Baekey, P. Skipper and E.M. Hadden, 1999. Interleukins and contrasuppression induce immune regression of head and neck cancer. Arch. Otolaryngol., 120: 395-403. http://www.ncbi.nlm.nih.gov/entrez/ query.fcgi? $\mathrm{cmd}=$ Retrieve $\& \mathrm{db}=$ PubMed $\& \mathrm{dopt}=\mathrm{Cit}$ ation\&list_uids $=8166968$.
2. Münz, C., 2004. Epstein-Barr Virus Nuclear Antigen 1: From immunologically invisible to a promising T cell target. J. Exp. Med., 199: 13011304. Doi: 0.1084/jem.20040730 http://www.ncbi. nlm.nih.gov/entrez/query.fcgi? $\mathrm{cmd}=$ Retrieve $\& \mathrm{db}=$ PubMed\&dopt $=$ Citation\&list_uids $=15148332$

3. Kieff, E., A. Rickinson, 2001. Epstein Barr virus and its Replication. In: Fields Virology (eds D.M. Knipe and P.M. Howley) pp: 2511-2573. Lippincott Williams and Wilkins, Philadelphia, Pa. ISBN-13: 978-0781718325. http://www.amazon.com/FieldsVirology-David-M-Knipe/dp/0781718325

4. Couch, M.E., R.L. Ferris, J.A. Brennan, W.M. Koch, E.M. Jaffee, M.S. Leibowitz, G.T. Nepom, H.A. Erlich and D. Sidransky, 2007. Alteration of cellular and humoral immunity by mutant p53 protein and processed mutant peptide in head and neck cancer. Clin. Cancer Res., 13: 99-206. http://www.ncbi.nlm.nih.gov/entrez/ query.fcgi? $\mathrm{cmd}=$ Retrieve $\& \mathrm{db}=$ PubMed\&dopt $=\mathrm{Cit}$ ation\&list_uids $=18056201$

5. Ben-Trivedi, A., N. Kitabatake and E. Doi, 2001. Toxicity of dimethyl sulfoxide as a solvent in bioassay system with $\mathrm{HeLa}$ cells evaluated colorimetrically with 3-(4,5-dimethylthiazol-2-yl)2,5-diphenyl-tetrazolium bromide. Agric. Biol. Chem., 54: 2961-1966. http://www.ncbi.nlm.nih. gov/entrez/query.fcgi? $\mathrm{cmd}=$ Retrieve $\& \mathrm{db}=\mathrm{PubMed}$ $\&$ dopt $=$ Citation\&list_uids $=1368650$

6. Alhamarneh, O., S.M. Amarnath, N.D. Stafford and J. Greenman, 2008. Regulatory T-cells: What role do they play in antitumor immunity in patients with head and neck cancer? Head and Neck, 30: 251-261. http://www.ncbi.nlm.nih.gov/entrez/ query.fcgi? $\mathrm{cmd}=$ Retrieve $\& \mathrm{db}=$ PubMed $\& \mathrm{dopt}=\mathrm{Cit}$ ation\&list_uids $=18172882$.

7. Pak, A.S., M.A. Wright, J.P. Matthews, S.L. Collins, G.J. Petruzzelli and M.R.I. Young, 1995. Mechanisms of immune suppression in patients with head and neck cancer: Presence of CD34 (+) cells which suppress immune functions within cancers that secrete granulocytemacrophage colony-stimulating factor. Clin. Cancer Res., 1: 95-103. http://www.ncbi.nlm.nih. gov/entrez/query.fcgi? $\mathrm{cmd}=$ Retrieve $\& \mathrm{db}=$ PubMed $\&$ dopt $=$ Citation\&list_uids $=9815891$.

8. Maison, J.M., G.G. Kitchens, R.J. Eastham and B.R. Jennings, 1977. T-lymphocytes and survival of head and neck squamous cell carcinoma. Arch. Otolar. Head Neck Surg., 103: 223-227. http://www.ncbi.nlm.nih.gov/entrez/query.fcgi?cm $\mathrm{d}=$ Retrieve $\& \mathrm{db}=$ PubMed\&dopt $=$ Citation\&list_uids $=300622$. 
9. Chang, M.C., C.P. Chiang, C.L. Lin, J.J. Lee, L.J. Hahn and J.H. Jeng, 2005. Cell-mediated immunity and head and neck cancer: With special emphasis on betel quid chewing habit. Oral Oncol., 41: 757-775. doi: 10.1016/j.oraloncology.2005.01. 007 . http://www.ncbi.nlm.nih.gov/entrez/query. fcgi? $\mathrm{cmd}=$ Retrieve $\& \mathrm{db}=$ PubMed\&dopt $=$ Citation $\&$ list_uids $=16109353$.

10. Moriniere, S., 2006. Epidemiology of head and neck cancer. Rev. Prat., 56: 1637-1641. http://www.ncbi.nlm.nih.gov/entrez/query.fcgi?cm $\mathrm{d}=$ Retrieve\&db=PubMed\&dopt=Citation\&list_uids $=17137247$.

11. Wahlstrom, J., M. Berlin, C.M. Skold, H. Wigzell, A. Eklund and J. Grunewald, 1999. Phenotypic analysis of lymphocytes and monocytes/macrophages in peripheral blood and bronchoalveolar lavage fluid from patients with pulmonary sarcoidosis. Thorax, 54: 339-346. http://www.ncbi.nlm.nih.gov/entrez/query.fcgi? $\mathrm{cm}$ $\mathrm{d}=$ Retrieve\& $\mathrm{db}=$ PubMed\&dopt $=$ Citation\&list_uids $=10092696$.

12. Shimoyama, Y., T. Kubota and M. Watanabe, 1989. Predictability of in vivo chemosensitivity by in vitro MTT assay with reference to colonogenic assay. J. Surg. Oncol., 41: 12-18. http://www.ncbi.nlm.nih.gov/entrez/query.fcgi? $\mathrm{cm}$ $\mathrm{d}=$ Retrieve $\& \mathrm{db}=$ PubMed\&dopt $=$ Citation\&list_uids $=2716329$.

13. Kane, M.D., R.D. Schwarz, L.St. Pierre, M.D. Watson, M.R. Emmerling, P.A. Boxer and G.K. Walker, 1999. Inhibitors of V-type ATPases, bafilomycin $\mathrm{A} 1$ and concanamycin $\mathrm{A}$, protect against beta-amyloid-mediated effects on 3-(4, 5dimethylthiazol-2-yl)-2, 5-diphenyltetrazolium bromide (MTT) reduction. J. Neurochem., 72: 1939-1947. http://www.ncbi.nlm.nih.gov/ entrez/query.fcgi? $\mathrm{cmd}=$ Retrieve $\& \mathrm{db}=$ PubMed $\&$ do $\mathrm{pt}=$ Citation\&list_uids $=10217271$.

14. Taylor, R.B., P.H. Duffus, M.C. Raff and S. De Petris, 1971. Redistribution and pinocytosis of lymphocyte surface immunoglobulin molecules induced by anti-immunoglobulin. Nat. New. Biol, 233: 225-229. http://chemport.cas.org/cgi$\mathrm{bin} / \mathrm{sdcgi}$ APP $=$ ftslink\&action=reflink\&origin $=\mathrm{np}$ g\&version $=1.0 \&$ coi $=1:$ CAS:528:DyaE38XntVyms Q\%3D\%3D\&pissn=0028-0836\&pyear $=1989 \&$ md5 $=979584 \mathrm{~b} 112 \mathrm{~b} 06 \mathrm{ebf} 32 \mathrm{e} 03406 \mathrm{cbb} 61 \mathrm{~d} 34$.

15. Bergler, W., G. Petroianu and A. Schadel, 1993. Feasibility of proliferation studies using the BrdU and MTT assays with a head and neck carcinoma cell line. ORL J.Otorhinolaryngol. Relat. Spec., 55: 230-235. http://www.ncbi.nlm.nih.gov/entrez/ query.fcgi? $\mathrm{cmd}=$ Retrieve $\& \mathrm{db}=$ PubMed $\&$ dopt $=\mathrm{Cit}$ ation\&list_uids $=8336924$
16. Wong, H.T., T.A. Shahrizal, N. Prepageran, W.K. Lim and R. Raman, 2007. Cell-mediated immunity in nasopharyngeal carcinoma and allergic rhinitis: A controlled study. Ear Nose Throat J., 86: 292-294. http://www.ncbi.nlm.nih. gov/entrez/query.fcgi? $\mathrm{cmd}=$ Retrieve\&db=PubMed $\&$ dopt $=$ Citation\&list_uids $=17580811$.

17. Moss, D.J., S.H. Chan, S.R. Burrows, T.S. Chew, R.G. Kane, J.A. Staples and N. Kunaratnan, 1983. Epstein Barr virus specific T-cell response in nasopharyngeal carcinoma patients. Int. Cancer, 32: 301-305. http://www.ncbi.nlm.nih.gov/entrez/ query.fcgi? $\mathrm{cmd}=$ Retrieve $\& \mathrm{db}=$ PubMed\&dopt $=\mathrm{Cit}$ ation\&list_uids $=6309678$.

18. Lapointe, H., H. Lampe and D. Banerjee, 1992. Head and neck squamous cell carcinoma cell lineinduced suppression of in vitro lymphocyte proliferative responses. Otolaryngology, 106: 149-158. http://www.ncbi.nlm.nih.gov/entrez/ query.fcgi? $\mathrm{cmd}=$ Retrieve $\& \mathrm{db}=$ PubMed\&dopt $=\mathrm{Cit}$ ation\&list_uids $=1738546$.

19. Young, M.R.I., M.A. Wright, Y. Lozano, J.P. Mathews, J. Benefield and M.M. Preche, 1996. Mechanisms of immune suppression in patients with head and neck cancer: Influence on the immune infiltrate of the cancer. Int. J. Cancer, 67: 333-338. Doi: 10.1002/(SICI)10970215(19960729)67:3<333::AID-IJC5>3.0.CO;2-S. http://www.ncbi.nlm.nih.gov/entrez/query.fcgi?cm $\mathrm{d}=$ Retrieve\& $\mathrm{db}=$ PubMed\&dopt $=$ Citation\&list_uids $=8707405$.

20. Ferris. R.L., T.L. Whiteside and S. Ferrone, 2006. Immune escape associated with functional defects in antigen-processing machinery in head and neck cancer. Clin. Cancer Res., 12: 3890-3895. Doi: 10.1158/1078-0432.CCR-05-2750. http://www. ncbi.nlm.nih.gov/entrez/query.fcgi?cmd=Retrieve $\& \mathrm{db}=$ PubMed\&dopt $=$ Citation\&list_uids $=1681868$ 3.

21. Lakhdar, M., R. Oueslati, R. Ellouze, H. Thameur, M. Cammoun, N. Khedhiri and R. Kastally, 1989. High interferon titer and deffective Nk-cells activity in the circulation of nasopharyngeal carcinoma patients. Int. J. Cancer, 43: 543-548. http://www.ncbi.nlm.nih.gov/entrez/query.fcgi?cm $\mathrm{d}=$ Retrieve $\& \mathrm{db}=$ PubMed\&dopt $=$ Citation\&list_uids $=2539326$.

22. Tsuchiya, S., 2002. Diagnosis of epstein-bar virusassociated diseases. Crit. Rev. Oncol. Hematol., 44: 227-238. Doi: S1040842802001142 http://www.ncbi.nlm.nih.gov/entrez/query.fcgi?cm $\mathrm{d}=$ Retrieve $\& \mathrm{db}=$ PubMed\&dopt $=$ Citation\&list_uids $=12467963$. 
23. Iwatsuki, K., T. Yamamoto, K. Tsuji, D. Suzuki, K. Fujii, H. Matsuura and T. Oono, 2004. A spectrum of clinical manifestations caused by host immune responses against epstein-barr virus infections. Acta Med. Okayama, 58: 169-180. http://www.ncbi.nlm.nih.gov/entrez/query.fcgi?cm $\mathrm{d}=$ Retrieve $\& \mathrm{db}=$ PubMed\&dopt $=$ Citation\&list_uids $=15551754$.
24. Mun-Hon, N.G., Chan Wwog-Hung, Sze Ng and Zong Yong, 2006. Epstein Ba rr virus serology in early detection and screening of nasopharyngeal carcinoma. Hinsess. J. Cancer, 25: 250-256. http://www.ncbi.nlm.nih.gov/entrez/query.fcgi?cm $\mathrm{d}=$ Retrieve\& $\mathrm{db}=$ PubMed\&dopt $=$ Citation\&list_uids $=16480597$ 